\title{
Diseño e Implementación de un Sistema Bifrecuencial Basado en Láseres de Baja Potencia
}

\author{
Matango, Santiago ${ }^{1}$ (i) ; Ávalos, Eduardo ${ }^{1 \text {,* }^{*}}$ \\ ${ }^{1}$ Escuela Politécnica Nacional, Facultad de Ingeniería Eléctrica y Electrónica, Quito, Ecuador
}

\begin{abstract}
Resumen: El presente trabajo describe el diseño e implementación de un sistema bifrecuencial que puede ser usado como alternativa para el tratamiento de enfermedades en la boca mediante la irradiación de luz láser de baja potencia. El sistema es un prototipo, cuyo diseño mecánico es creado en un software de uso libre para luego ser impreso en un modelo 3D el cual alberga toda la parte electrónica y óptica. En cuanto a la parte electrónica, se monta sobre varios módulos los cuales cumplen diferentes funciones como son: el cargador de la batería, convertidores de potencia, drivers para los disparadores, una pantalla OLED que muestra información de los sensores, todo el proceso y mandos tanto manuales como automáticos son controlados por una tarjeta de desarrollo. El sistema óptico comprende de; un arreglo de espejos que básicamente dan el direccionamiento a la luz de los láseres y hacen que incidan sobre un mismo punto, al final se integra una fibra óptica para poder ingresar a partes inaccesibles en la boca para el tratamiento. Para validar el prototipo implementado, se realizaron mediciones tanto de la irradiancia como de la potencia en equipos especializados para estas aplicaciones, a más de basarse en revistas odontológicas y artículos de salud publicados por distintos institutos y universidades.
\end{abstract}

Palabras clave: Láser, bifrecuencial, espejos, tarjeta de desarrollo, irradiancia.

\section{Design and Implementation of a Bifrequencial System Based on Low Power Lasers}

\begin{abstract}
This paper describes the design and implementation of a bifrequencial system that can be used as an alternative for the treatment of diseases in the mouth by irradiating low power laser light. The system is a prototype, whose mechanical design is created in a free-use software and then printed on a 3D model which houses all the electronic and optical part, as for the electronic part it is mounted on several modules which meet different functions such as the battery charger, power converters, drivers for the triggers, an OLED screen that displays sensor information, the entire process and both manual and automatic controls are controlled by a development card. The optical system comprises an arrangement of mirrors that basically, gives the light direction of the lasers and make them impact on the same point, in the end an optical fiber is integrated to be able to enter inaccessible parts in the mouth for treatment. To validate the implemented prototype, measurements were made of both irradiance and power in specialized equipment for these applications, in addition to being based on dental journals and health articles published by different institutes and universities.
\end{abstract}

Keywords: Laser, bifrequencial, mirrors, development card, irradiance.

\section{INTRODUCCIÓN}

Las enfermedades de la boca son lesiones que se presentan como úlceras causando inflamación del tejido blando, las cuales pueden originarse por agentes mecánicos, químicos, biológicos y endógenos del organismo, estas se manifiestan de manera aguda, crónica o recurrente. Una de las lesiones de este tipo, cuyo comportamiento es reincidente son las aftas bucales. El afta es una lesión que se considera multifactorial, aunque en la actualidad sus causas no están del todo definidas, se caracterizan por la aparición de úlceras dolorosas las cuales son variables en forma y tamaño. Otra enfermedad que es muy común en la población es el herpes simple tipo 1, el cual se presenta como úlceras bucales o ampollas febriles causando llagas alrededor de la boca o en el rostro, no existe ningún tratamiento que elimine por completo el virus que la causa, solo existen tratamientos que hacen que el virus se inactive pero ante cualquier cambio ambiental o corporal el virus volverá a aparecer (España, y otros, 2004). Es así como las llagas que se pueden desarrollar en la boca o a su alrededor se han convertido en un problema muy doloroso y molestoso para las personas, lesiones que pueden durar varios días en desaparecer dejando en algunos casos marcas de alguna cicatriz a la persona que lo padece. 
Existen varios tipos de radiación laser, clasificadas según su potencia o energía en alta, media y los denominados baja potencia, también conocidos como láseres blandos (soft-laser o terapéuticos), por el efecto fotoquímico que predomina en él, ya que al incidir el haz de luz sobre la piel o mucosa, se deposita una gran cantidad de energía que provoca a nivel celular un desencadenamiento de reacciones bioquímicas, bioeléctricas y bioenergéticas (Zaldívar, y otros, 1997) que logran producir un efecto analgésico, antiinflamatorio y bioestimulante, según la dosis de energía utilizada, en la Tabla 1 (España, y otros, 2004), se muestra los distintos láseres que son utilizados en medicina para diferentes tratamientos de enfermedades, a los cuales se los asigna un valor numérico que corresponde al uso de cada laser; es decir: $0=$ contraindicado, $1=$ se puede utilizar, $2=$ indicado, $3=$ bastante indicado y $4=$ muy indicado.

Tabla 1. Tratamientos en terapéutica dental.

\begin{tabular}{llllll}
\hline Tratamiento/Láser & Diodo & Nd:YAG & Er,Cr:YSGG & Er:YAG & CO2 \\
\hline $\begin{array}{l}\text { Preparación de } \\
\text { cavidades }\end{array}$ & 0 & 1 & 4 & 4 & 0 \\
$\begin{array}{l}\text { eliminación de } \\
\text { composites }\end{array}$ & 0 & 0 & 4 & 4 & 0 \\
$\begin{array}{l}\text { Grado de esmalte } \\
\text { Sellado de fosas y }\end{array}$ & 0 & 1 & 4 & 4 & 2 \\
$\begin{array}{l}\text { fisuras } \\
\text { Hiperestesia }\end{array}$ & 1 & 2 & 4 & 4 & 1 \\
$\begin{array}{l}\text { dentinaria } \\
\text { Endodoncia }\end{array}$ & 1 & 2 & 4 & 4 & 2 \\
$\begin{array}{l}\text { Carillas estéticas } \\
\text { Blanqueamiento }\end{array}$ & 0 & 4 & 2 & 2 & 1 \\
$\begin{array}{l}\text { dental } \\
\text { Preparación de }\end{array}$ & 4 & 4 & 0 & 4 & 0 \\
coronas & 0 & 0 & 3 & 0 & 1 \\
\hline
\end{tabular}

El tratamiento con luz láser resulta ser una técnica no invasiva, efectiva y menos doloroso que otros tratamientos tradicionales ya que la luz actúa de manera directa al penetrar la piel. La luz es absorbida por los cromóforos (sustancias que tiene electrones), es decir la energía de la luz es transformada a energía útil desencadenando una serie de procesos bioquímicos como es el aumento de oxígeno y liberación de óxido nítrico en las células afectadas por los virus, dando como resultado la reducción del dolor, inflamación y acelera el metabolismo intracelular (Pacheco, y otros, 2014).

Los últimos avances en mecanismos tecnológicos en el tratamiento con láser han hecho que cada vez sea más factible usarlo en diversas áreas de la medicina, ya que el tejido humano transmite la luz roja de manera eficiente, en donde la profundidad de penetración de la luz dependerá de la longitud de onda y dosificación adecuada. La profundidad que alcanza la luz varía en cada tejido, al igual que el tiempo de la aplicación de la misma que puede durar entre 30 a 90 segundos aproximadamente (Stolik, y otros, 2000) (Tutivén, 2017).

La irradiación representa la cantidad de energía acumulada en el tiempo que incide en una área, por ejemplo tenemos la irradiación solar que cae sobre la superficie de la tierra la cual esta expresada en kilovatio hora ó kiloJoule por metro cuadrado $\left(\mathrm{kWh} / \mathrm{m}^{2}\right.$ ó $\left.\mathrm{kJ} / \mathrm{m}^{2}\right)$ para un valor constante de sol (Ponce, 2018). De la misma manera se mide la irradiación de un haz de luz láser al incidir sobre la piel, el cual es un parámetro fundamental para poder producir efectos bioquímicos en las células afectadas y estas transformen la energía irradiada por medio de los cromóforos para cerrar vasos sanguíneos y evitar que la sensación de dolor se propague al sistema nervioso, por otra parte se tiene que tener en cuenta que no debe ser elevada puesto que podría causar daños en el tejido ni tampoco debe ser baja ya que los cromóforos no reaccionarían (Stolik, 2000).

La expresión (1) muestra la ecuación general de la irradiación, donde el flujo irradiante (E) es energía emitida o reflejada por una superficie $\left(\mathrm{W} / \mathrm{cm}^{2}\right)$ acumulado en un intervalo de tiempo (s).

$$
D=E \cdot t\left[\frac{J}{c m^{2}}\right]
$$

En (Marotti, 2009), se presenta un artículo donde se hace el tratamiento de un herpes simple labial con luz láser de baja intensidad varias personas, con un equipo comercial desarrollado en Brasil, el cual muestra un resultado muy bueno en la zona afectada, las lesiones fueron irradiadas con el láser dúo a una longitud de onda de $660 \mathrm{~nm}$ en modo continuo y una energía de $120\left(\mathrm{~J} / \mathrm{cm}^{2}\right), 40(\mathrm{~mW})$ durante 2 minutos. El tratamiento se lo repitió cada 24 horas, después de 72 horas y 1 semana. Se muestran los resultados esperados en la estética de los labios, como se presenta en la Figura 1.
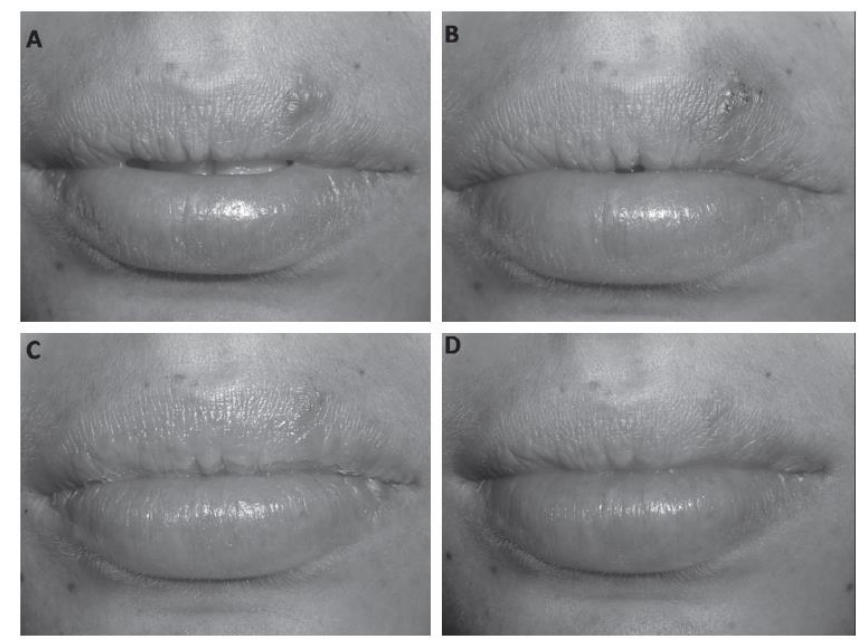

Figura 1. A) Lesión antes del tratamiento con láser, B) Misma lesión después de aplicar el láser, C) Lesión después de 24 horas de tratamiento, D) Una semana después.

En (Hernández, y otros, 2013) se toma un grupo de personas que padecen de aftas bucales $(\mathrm{N}=90)$, pacientes mayores de 5 años de edad y que desearon participar en la investigación, a este grupo se lo dividió en grupo A y B de a 45 pacientes cada uno, al grupo A se le aplico terapia laser con parámetros analgésicos, antiinflamatorios durante 7 días, con una potencia de $30 \mathrm{~mW}$, en un tiempo de $36 \mathrm{seg}$, y una dosificación de 1-3 $\mathrm{J} / \mathrm{cm} 2$, el equipo comercial utilizado fue uno de fabricación cubana LASERMED 670 DL, que utiliza como emisor un diodo laser de arseniuro de galio y aluminio (GA As AL), con una longitud de onda de $670 \mathrm{~nm}$.

Al grupo B se lo trato con tintura de propóleo al 5\% (medicamento local), con una frecuencia diaria hasta los 7 días de tratamiento. Mas de la mitad de los pacientes tratados con terapia laser eliminaron y redujeron el dolor en comparación con el otro grupo.

El presente trabajo pretende desarrollar un prototipo portátil para ser usado como alternativa en odontología, contiene dos láseres de baja potencia, láser rojo $(660 \mathrm{~nm})$ y láser infrarrojo (808nm) los cuales se activarán de manera alternada 
dependiendo del problema a tratar, mediante un arreglo de espejos y lentes se direcciona la luz a un punto para luego ser transportada por una fibra óptica, de esta manera la luz es dirigida fácilmente a la llaga bucal. La electrónica implementada será la encargada de proveer la potencia y energía necesaria para el tratamiento de enfermedades que aparecen en la boca.

\section{CONCEPTOS FUNDAMENTALES}

\subsection{Beneficios de la luz en la piel}

Según la teoría de mecánica cuántica, la energía de la luz está compuesta de fotones o paquetes discretos de energía electromagnética. La energía de un fotón depende solo de la longitud de onda, por tal razón la energía de una dosis de luz depende solo de la cantidad de fotones y de su longitud de onda. Los fotones que se envían al tejido vivo pueden ser absorbidos o dispersados, los fotones dispersados escapan del tejido en forma de reflexión difusa, mientras que los fotones absorbidos interactúan con una molécula orgánica o cromóforo ubicado dentro del tejido. Debido a que estos fotones tienen longitudes de onda en las regiones roja e infrarroja, los cromóforos que absorben estos fotones tienden a tener electrones que son excitados por la cantidad de energía que entrega el fotón (Pacheco, 2014). De acuerdo con la primera ley de termodinámica, la energía entregada al tejido debe conservarse, lo que ocurre cuando la luz es absorbida por el tejido vivo se llama conversión interna, esto sucede cuando el primer estado excitado del cromóforo sufre una transición de un estado electrónico alto a uno bajo (Núñez, 2014). La energía del estado excitado se emite en forma de calor, la energía entregada al tejido puede ser transformada en fluorescencia. La fluorescencia es una luminiscencia en la cual la absorción molecular de un fotón desencadena la emisión de otro fotón con una longitud de onda más larga, la diferencia de energía entre los fotones absorbidos y emitidos termina como calor.

El tejido cumple con dos características ópticas importantes, como son: la absorción y la penetración. La energía que llevan los fotones al ser absorbidos por los cromóforos (sustancias que contienen electrones) es transformada a energía útil desencadenando una serie de procesos bioquímicos como es el aumento de oxígeno y liberación de óxido nítrico en las células afectadas, dando como resultado la reducción del dolor, inflamación y acelera el metabolismo intracelular (Carrasco, y otros, 2018). Existen tres áreas de la medicina en las que la luz láser juega un papel importante como se muestra en la Figura 2, estos son: cicatrización de heridas y reparación de tejidos, alivio de la inflamación en enfermedades crónicas y lesiones, alivio del dolor neurogénico y algunos problemas neurológicos (Calabrese, 2011).

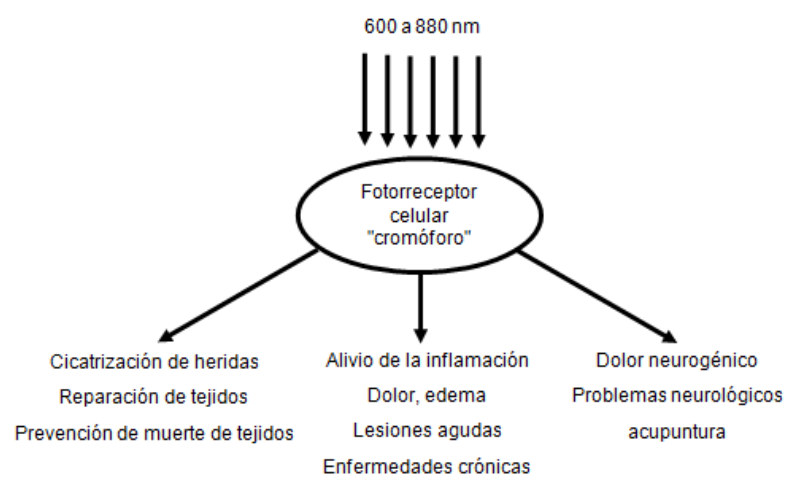

Figura 2. Representación esquemática de las principales áreas de aplicación con luz en el espectro rojo e infrarrojo.

Hay que considerar las propiedades ópticas del tejido, ya que tanto la absorción como la dispersión de la luz en el tejido dependen de la longitud de onda y el cromóforo tisular principal (hemoglobina y melanina) tiene bandas de absorción a altas en longitudes de onda cortas inferiores a los $600 \mathrm{~nm}$, el agua comienza a absorberse a longitudes de onda superiores a $1150 \mathrm{~nm}$, por tal razón se presenta un ventana óptica la cual se observa en la Figura 3, en donde las longitudes de onda roja e infrarroja maximizan la penetración efectiva de la luz en el tejido (Hamblin, y otros, 2013).

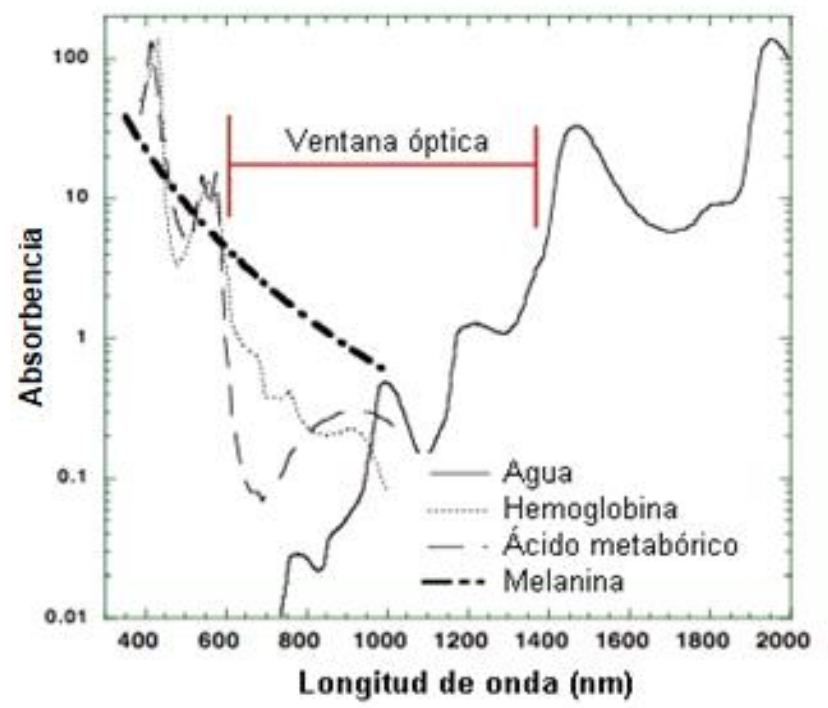

Figura 3. Ventana óptica en el tejido debido a la absorción de longitudes de onda roja e infrarroja por los cromóforos.

\subsection{Estudios clínicos}

La terapia con láser de baja potencia se usa para tratar una gran variedad de enfermedades y dolores musculares agudos y crónicos, por ejemplo: los dentistas lo usan para tratar los tejidos orales inflamados y curar diversas úlceras, los dermatólogos para tratar acné, manchas en la piel y quemaduras, los ortopedistas lo usan para aliviar el dolor y tratar inflamaciones crónicas. La luz generada por láseres y LEDs se aplica directamente a las áreas a tratar, por ejemplo: heridas, lesiones, úlceras, o en varios puntos del cuerpo, por ejemplo: puntos de acupuntura, puntos de activación muscular. Las aplicaciones clínicas de la terapia con luz de baja potencia son diversas, su uso se caracteriza por una variedad de metodologías para las diversos láseres y LEDs con sus especificaciones como: longitud de onda, potencia de salida, modos de operación (onda continua o pulsada) y parámetros 
de pulsos. En los últimos años se ha preferido longitudes de onda largas (650 a $880 \mathrm{~nm})$ y potencias de salida altas (hasta $100 \mathrm{~mW}$ ) en dispositivos terapéuticos, especialmente para permitir una penetración tisular profunda (Hamblin, 2013).

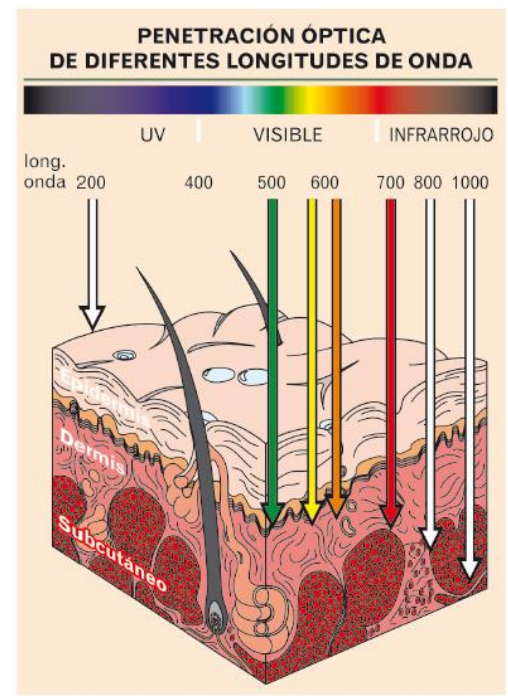

Figura 4. Penetración de la luz en la piel.

\subsection{Enfermedades de la boca}

Muchas lesiones en el tejido de la boca se relacionan con problemas anatómicos, funciones del organismo y otras aparecen como reacciones que generalmente son de origen nutricional e inmunológico, muchas de las cuales son difíciles de tratar con medicina tradicional, por lo general las aftas, llagas y úlceras que aparecen con frecuencia se relacionan más con enfermedades digestivas. El desarrollo de un afta suele prolongarse por días y pasa por varios periodos hasta desaparecer, proceso que es muy doloroso y con sensación de quemazón, son ovaladas, de color blanquecino rodeadas por una corona rojiza, las aftas pueden surgir en cualquier parte de la vía oral como se muestra en la Figura 5. (Pacho, y otros, 2006).

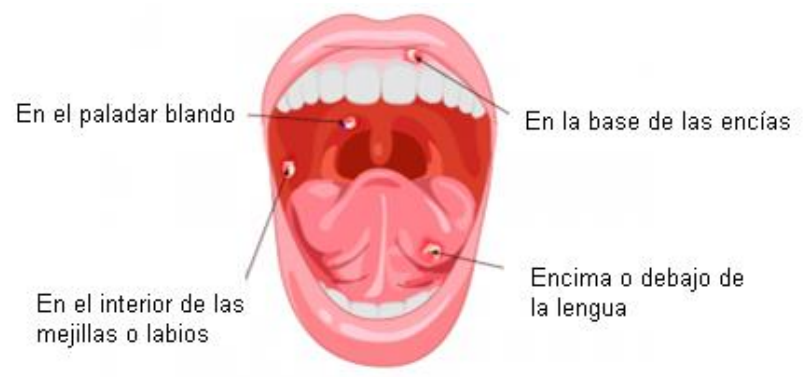

Figura 5. Zonas de la boca en que se desarrollan las aftas.

El herpes labial es otro problema de salud bucal que afecta aproximadamente un tercio de la población, causado por Herpes virus hominis el cual se transmite por saliva infectada, sin embargo, algunas personas pueden tener este virus sin haber desarrollado ningún síntoma, al estar presente en el tejido blando de la boca produce una afección eruptiva aguda de uno o más grupos de vesículas, causando lesiones dentro de la boca para contrarrestar estos virus el organismo desarrolla anticuerpos. Causa frecuentes episodios de malestar, dolor y estética, los medicamentos antivirales no han tenido éxito en la eliminación total del virus (de Paula, y otros, 2013) (González, y otros, 2008).

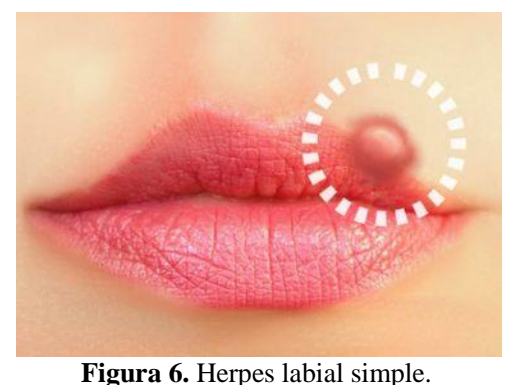

En avance tecnológico se han hecho un sinnúmero de estudios relacionados a la luz láser usado para tratamientos de enfermedades de la vía oral, obteniendo buenos resultados en el proceso, el láser al producir calor en la zona irradiada hace que suceda una reacción bioquímica en las células afectadas, la luz es absorbida por los cromóforos en la cadena respiratoria de las mitocondrias, dando lugar a cambios fundamentales, como el aumento de oxígeno y liberación de óxido nítrico. La estimulación nerviosa mediante un láser altera la hiperpolarización de la membrana celular y aumento de la concentración de ATP (nucleotido), que contribuye a mantener la estabilidad de la membrana y disminuyendo el umbral de dolor (Schindl, y otros, 1999).

\subsection{Tratamiento de enfermedades bucales con láser}

El tratamiento con láser implica un gran número de parámetros en ciertos casos se suele usar un fotosensibilizador con absorción resonante por la fuente de luz. Las fuentes de luz más utilizadas son los láseres rojos e infrarrojos, debido a sus ventajas como es producir una sola longitud de onda y la dosimetría de la luz adecuada es fácil de calcular, al final el rayo láser puede pasar a través de una fibra óptica para tratamiento localizado. Es así que la radiación con luz láser cumple el objetivo de tratar ciertas enfermedades bucales irradiando la zona afectada, ocasiona cambios físicos y químicos denominados procesos fotobiológicos los cuales se los usan ventajosamente en combatir enfermedades de la piel (Marotti, y otros, 2009). La radiación con láser de baja potencias, desencadena una serie de reacciones bioquímicas, biogenéticas y bioeléctricas a nivel celular, lo que reduce la sensación de dolor, edema y estimula la regeneración tisular (tejido afectado) (Pacheco, 2013) (Valiente, y otros, 1997).

\section{METODOLOGÍA}

El proyecto al ser un primer estudio se emplea la investigación aplicada, ya que con el desarrollo de este prototipo se espera dar una solución alternativa al uso de tratamientos convencionales como: pomadas o soluciones usadas para enfermedades que se presentan en la boca o sus alrededores. El tratamiento no invasivo se lo realiza mediante la irradiación con luz láser de baja potencia. Para lograrlo, se realizó la recopilación de fuentes bibliográficas como: libros, revistas médicas, artículos científicos y páginas web.

En la Figura 7, se muestra la estructura general del prototipo el cual se compone de cuatro sistemas que son esenciales para el funcionamiento y un fácil manejo de usuario, los cuales se describen a continuación. 


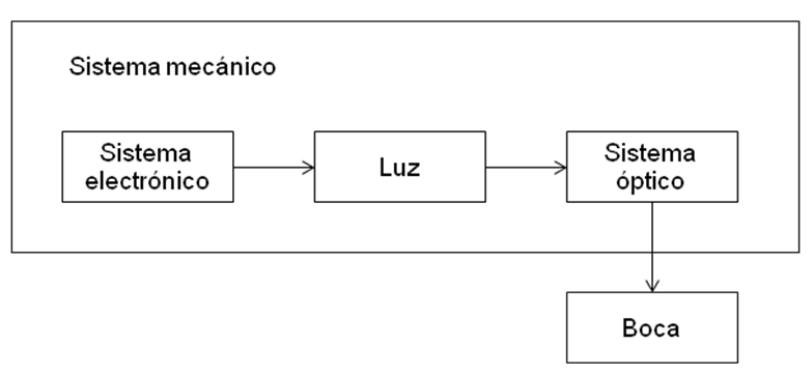

Figura 7. Esquema general del sistema bifrecuencial.

El sistema electrónico es el encargado de suministrar la potencia eléctrica necesaria a cada componente digital o análogo, con el objetivo de tener un buen control de la irradiación producida por cada uno de los láseres. Cuenta con una pantalla OLED que permite interactuar con el usuario, en pantalla se muestran variables importantes del sistema como es el porcentaje de batería, menús, energía aplicada por cada láser y mensajes de batería baja o carga completa, los mensajes se acoplan a un LED de notificaciones mediante una salida digital de la tarjeta de desarrollo para hacerlos más perceptibles.

El sistema óptico es el encargado de direccionar la luz generada por los láseres hacia un mismo punto mediante un arreglo de espejos, la luz direccionada pasa por una fibra óptica que se usa en equipos odontológicos para ser irradiada en la zona a tratar.

El haz de luz que se irradia en la zona de muestra es generado por láseres de baja potencias a dos longitudes de onda diferentes las cuales están en el espectro de emisión de $660 \mathrm{~nm}$ y $808 \mathrm{~nm}$, luz roja e infrarroja respectivamente, activándose uno a la vez dependiendo del problema a tratar.

Finalmente, el sistema mecánico da soporte a todos los componentes que constituyen al prototipo como son: los espejos, los láseres, el circuito electrónico y la pantalla de visualización. Cuenta con un diseño de fácil maniobrabilidad y ergonomía.

\subsection{Sistema óptico}

Al ser el prototipo un sistema bifrecuencial se tiene que direccionar la luz de los láseres hacia un mismo punto mediante un arreglo de un espejo y un espejo dicroico para luego ser dirigida por una fibra óptica comercial usada en odontología, en la Figura 8. se muestra el direccionamiento de la luz hacia un mismo punto.

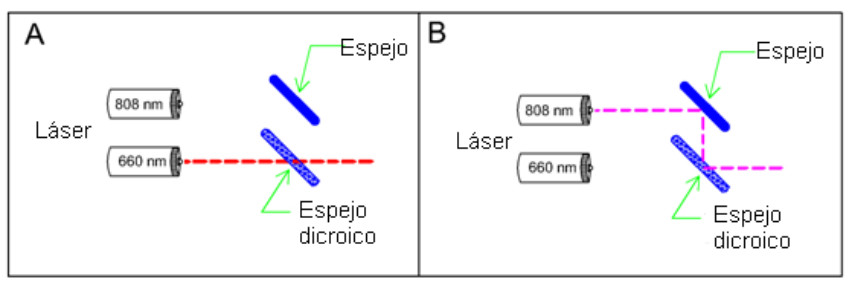

Figura 8. Trayectoria de los haces de luz.

La luz roja $(660 \mathrm{~nm})$ pasa el espejo dicroico para ser concentrada en un punto como se puede ver en la Figura 8.A, mientras que la luz infrarroja $(808 \mathrm{~nm})$ primero es reflejada en el espejo después se refleja nuevamente en el espejo dicroico para concéntrense en el mismo punto donde incide la luz roja, como se observa en la Figura 8.B. El objetivo de concentrar las luces en un punto es para que toda la luz sea dirigida hacia una fibra óptica y está la conduzca hacia la zona a irradiar.

\subsection{Sistema electrónico}

El principal objetivo del sistema electrónico es el control de la irradiación producido por las fuentes láser, compuesto por módulos que permiten la alergización de los distintos elementos presentes en la placa electrónica. Este sistema electrónico se conforma del sistema de potencia y de control, los cuales están aislados para evitar fallos y seguridad del sistema en general.

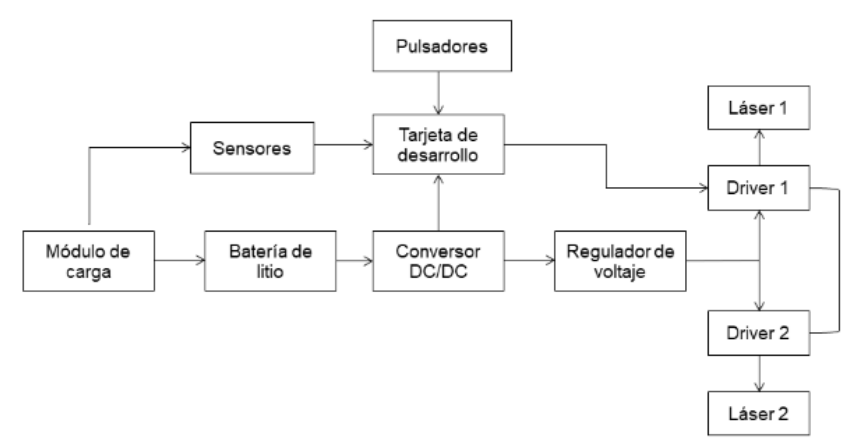

Figura 9. Diagrama en bloques del sistema electrónico general.

El sistema electrónico de potencia inicia con el módulo de carga, el cual es utilizado para carga y protección de la batería, la batería de litio es la encargada de suministrar energía a todo el sistema, la energía almacenada por la batería es tomada directamente por el conversor DC/DC para elevarla a un voltaje mayor al de la batería para poder energizar los demás elementos como los láseres, sensores y tarjeta de desarrollo.

El sistema electrónico de control está conformado por una tarjeta de desarrollo el cual integra un microcontrolador en su estructura como elemento central de control, es encargado de controlar los actuadores y procesar las señales analógicas y digitales captadas por los puertos de entrada y salida.

\subsection{Sistema electrónico}

La función del sistema mecánico es albergar la placa PCB y los componentes ópticos, a más de acoplar la fibra al sistema óptico, el diseño mecánico se lo realiza en el software de ingeniería y modelado SolidWorks muy usado a nivel mundial en industrias manufactureras, automotrices y robótica.

SolidWorks presenta una interfaz amigable y entendible donde las piezas se crean a partir de los dibujos o ensamblajes, además se puede importar la placa PCB simulada en Proteus para verificar que las dimensiones son las correctas para el prototipo, el sistema mecánico consta de tres partes como son: el soporte de los lentes y de los láseres, los cuales son sujetados y fijados por la carcasa hecho en plástico (PLA) en la Figura 10 se muestra el resultado final. 


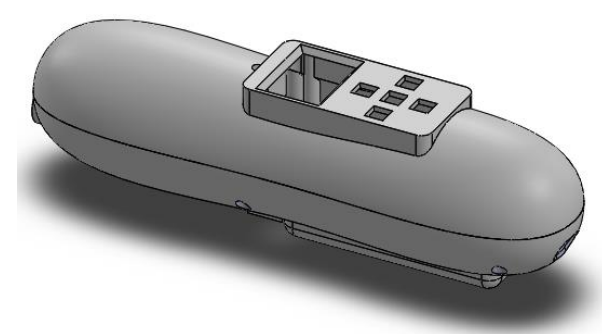

Figura 10. Carcasa diseñada.

\subsection{Hardware del sistema}

El diseño del sistema electrónico se lo hace en Proteus, el cual es un software conformado por dos aplicaciones Isis y Ares, los cuales permiten de manera interactiva la simulación de circuitos electrónicos y diseño de pistas PCB, en su paquete de instalación se encuentran librerías de componentes electrónicos pasivos o activos de todos los fabricantes que existen en el mercado de los circuitos integrados, también permite cargar a los microcontroladores presentes en sus librerías con los programas previamente realizados en los ensambladores y compiladores según sea el lenguaje de programación elegido (tecnicaslomas.com, 2019).

La placa PCB se la realiza a doble lado, de esta manera reducir el tamaño y aprovechar mejor el espacio, en un lado se colocan todos los módulos, en el otro la tarjeta de desarrollo y demás elementos e integrados como se ve en la Figura11, con dimensiones de $40 \mathrm{~mm}$ x $82.5 \mathrm{~mm}$, por seguridad se usa conectores tipo Molex para las conexiones.

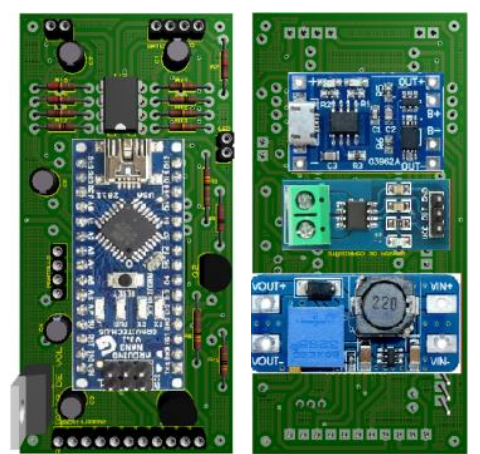

Figura 11. Placa PCB principal realizada a doble lado.

Para la parte de los botones que se integran al prototipo se realizó otra PCB de $32.5 \mathrm{~mm}$ x $30 \mathrm{~mm}$ para poder acoplarle a la carcasa. La PCB de los botones se muestra en la Figura 12.

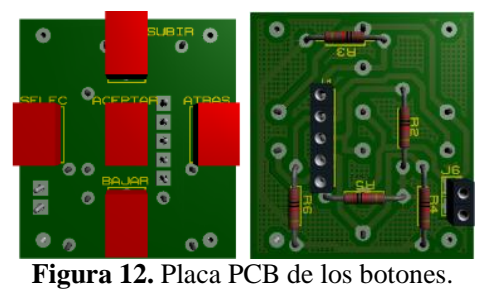

\subsection{Software del sistema}

El desarrollo del software de control se lo realiza en lenguaje de programación de Arduino IDE, debido a que ofrece un entorno de programación amigable mediante el uso de librerías que se encuentran en el paquete de instalación o que se las puede descargar de internet para luego ser compilada en la tarjeta de desarrollo.

En la Figura 13, se presenta el diagrama de entradas y salidas utilizado en la tarjeta de desarrollo, las cuales son programadas para recibir señales de voltaje generadas por los sensores, esta información es procesada y enviada mediante comunicación a dos hilos para ser visualizarla en forma de caracteres en pantalla y notificaciones visuales. La tarjeta de desarrollo envía señales de control que aumentan o disminuyen la intensidad de luz mediante pulsadores que se integran a las entradas digitales creando una interacción fácil y accesible para el usuario final.

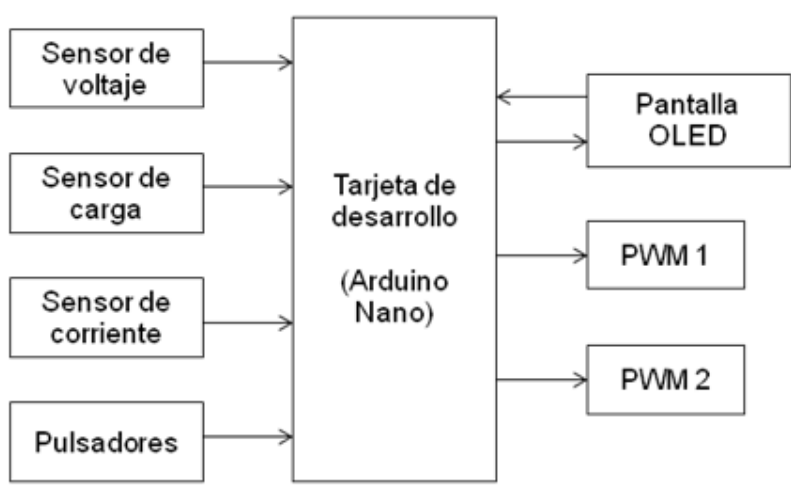

Figura 13. Diagrama de entradas y salidas de la tarjeta de desarrollo.

En el monitoreo de la batería los sensores son los encargados de medir el porcentaje de batería, si se conecta el cargador o si está cargado completamente, cuidando siempre que no se pase de los límites de carga 4.2 (V) y de descarga 2.8 (V), en la Figura 14, se presenta el diagrama de flujo de carga y descarga de la batería.

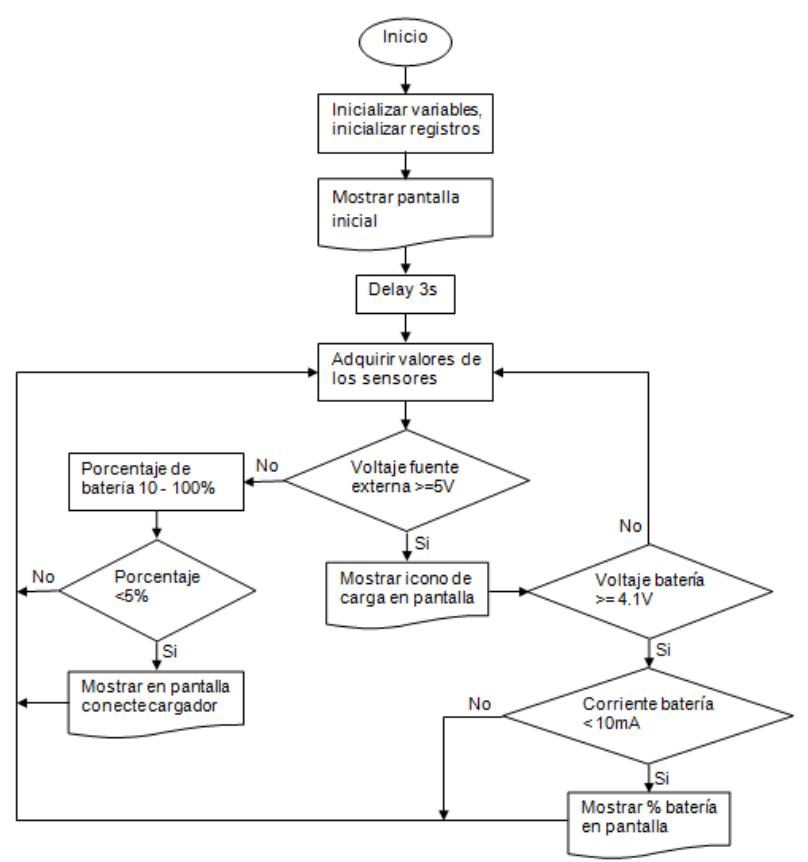

Figura 14. Diagrama de flujo para monitoreo de la batería. 


\subsection{Resultados}

Las pruebas que se realizan al equipo para la verificación del funcionamiento del equipo son: autonomía del equipo, sistema óptico y pruebas térmicas. Todas estas pruebas se las hizo basándose en las normas InMetro de Brasil y artículos publicados.

\subsubsection{Pruebas de autonomía del equipo}

La autonomía del prototipo se la calcula midiendo la corriente de consumo al estar siendo usado el láser a diferentes intensidades para lo cual se utiliza un multímetro ProsKit MT1270, las mediciones tomadas se encuentran en la Tabla 2, la Figura 15 muestra la medición tomada al estar operativo el láser rojo a la máxima intensidad.

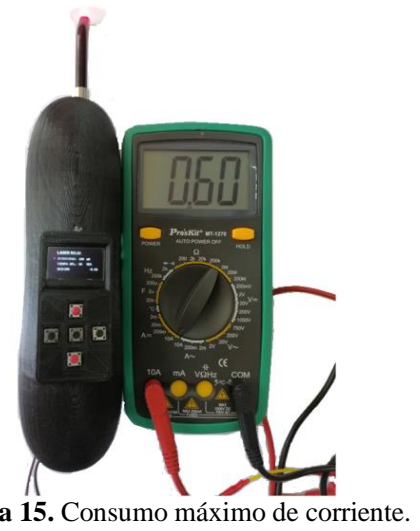

Teniendo en cuenta la capacidad de la batería KeepPower de $2600 \mathrm{mAh}$, se tiene un tiempo aproximado de autonomía del prototipo que se la calcula mediante la ecuación (2), los resultados se encuentran en la Tabla 2 y Tabla 3.

$$
t=\frac{2600 m A h}{I}
$$

Donde $(t)$ es el tiempo de autonomía en horas e (I) es la corriente de consumo expresada en mili-amperios.

Tabla 2. Resultados obtenidos del láser rojo.

\begin{tabular}{ccc}
\hline Iluminación [\%] & Corriente [mA] & Tiempo de autonomía [hrs] \\
\hline 0 & 150 & 17.33 \\
20 & 250 & 10.42 \\
40 & 350 & 7.42 \\
60 & 470 & 5.53 \\
80 & 560 & 4.64 \\
100 & 600 & 4.33 \\
\hline
\end{tabular}

Tabla 3. Resultados obtenidos del láser infrarrojo.

\begin{tabular}{ccc}
\hline Iluminación [\%] & Corriente [mA] & Tiempo de autonomía [hrs] \\
\hline 0 & 150 & 17.33 \\
20 & 190 & 13.68 \\
40 & 270 & 9.62 \\
60 & 350 & 7.52 \\
80 & 430 & 6.05 \\
100 & 480 & 5.41 \\
\hline
\end{tabular}

Al ser un sistema bifrecuencial se debe tener en cuenta que se enciende un láser a la vez dependiendo del tipo de problema a tratar, entonces de las Tablas 2 y 3 se tienen dos tiempos de autonomía, uno de 4.33 horas si únicamente se le tiene funcionando al láser rojo a su máxima intensidad y otros de 5.41 horas si únicamente funciona el láser infrarrojo a su máxima intensidad, estos dos tiempos son suficientes para el tratamiento fotodinámico ya que los tiempos de aplicación no van más allá de los tres minutos.

\subsubsection{Pruebas de irradiancia}

Las pruebas de irradiancia se las realiza de acuerdo con la Norma InMetro 8381 (inmetro.gov.br, 2019), con la ayuda de un radiómetro DeltaOhm HD 2102.2, el cual es un instrumento portátil con visualización LCD que mide la irradiancia en las regiones espectrales VIS-NIR, UVA, UVB y UVC. Para la medición de la irradiancia cuenta con una sonda la cual una vez conectada es reconocida automáticamente por el equipo, este instrumento tiene una función adicional que es la integral en el tiempo; es decir, calcula la densidad de energía (irradiación). Los datos son almacenados en la memoria interna del equipo los cuales se pueden transmitir directamente en tiempo real a una PC conectado al instrumento mediante un puerto USB 2.0 (DeltaOHM, 2019). En la Figura 16 se muestra la sonda LP 471 RAD conectada al radiómetro tomando muestras de la irradiancia y densidad de energía del láser rojo.

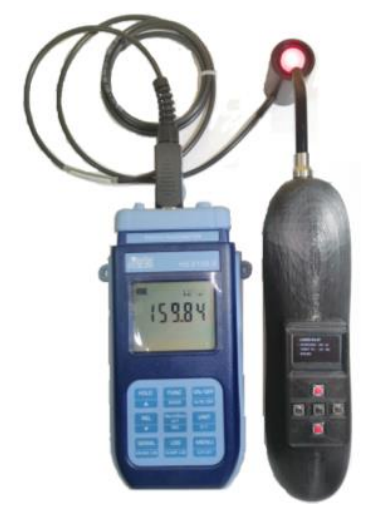

Figura 16. Pruebas de irradiancia e irradiación.

Se realizaron cinco pruebas, a diferentes niveles de potencia con una separación de $5 \mathrm{~mm}$ a la sonda a un tiempo máximo de 3 minutos por aplicación, el área irradiada tiene un diámetro de $8(\mathrm{~mm})$. Los resultados se encuentran en las Tablas 4 y 5 , con la ecuación (3) se calcula la energía en cada prueba.

$$
E=P . t
$$

Donde $(E)$ es la energía, $(P)$ es la potencia aplicada en Watt y $t$ es el tiempo de aplicación expresado en segundos.

Tabla 4. Datos de irradiancia e irradiación del láser rojo.

\begin{tabular}{ccccc}
\hline $\begin{array}{c}\text { Nivel } \\
{[\boldsymbol{m} \boldsymbol{W}]}\end{array}$ & $\begin{array}{c}\text { Irradiancia } \\
{\left[\mathbf{m W} / \mathbf{c m}^{2}\right]}\end{array}$ & $\begin{array}{c}\text { Tiempo } \\
{[\mathbf{s e g}]}\end{array}$ & $\begin{array}{c}\text { energía } \\
{[\boldsymbol{J}]}\end{array}$ & $\begin{array}{c}\text { irradiación } \\
{\left[\mathbf{J} / \mathbf{c m}^{\mathbf{2}}\right]}\end{array}$ \\
\hline 20 & 4.6 & 180 & 3.6 & 0.81 \\
40 & 9.5 & 180 & 7.2 & 1.22 \\
60 & 14.8 & 180 & 10.8 & 2.52 \\
80 & 20 & 180 & 14.4 & 3.52 \\
100 & 25.5 & 180 & 18 & 4.11 \\
\hline
\end{tabular}

Tabla 5. Datos de irradiancia e irradiación del láser infrarrojo.

\begin{tabular}{ccccc}
\hline $\begin{array}{c}\text { Nivel } \\
{[\boldsymbol{m} \boldsymbol{W}]}\end{array}$ & $\begin{array}{c}\text { Irradiancia } \\
{\left[\mathbf{m W} / \mathbf{c m}^{\mathbf{2}}\right]}\end{array}$ & $\begin{array}{c}\text { Tiempo } \\
{[\mathbf{s e g}]}\end{array}$ & $\begin{array}{c}\text { energía } \\
{[\boldsymbol{J}]}\end{array}$ & $\begin{array}{c}\text { irradiación } \\
{\left[\mathbf{J} / \mathbf{c m}^{\mathbf{2}}\right]}\end{array}$ \\
\hline 20 & 1 & 180 & 3.6 & 0.11 \\
40 & 3 & 180 & 7.2 & 0.42 \\
60 & 7.2 & 180 & 10.8 & 1.13 \\
80 & 12 & 180 & 14.4 & 1.83 \\
100 & 15 & 180 & 18 & 2.36 \\
\hline
\end{tabular}


Los resultados obtenidos en la Tabla 4 y Tabla 5 se los comparan con estudios similares publicados en revistas médicas de equipos usados en el tratamiento con luz para enfermedades de la boca, cuyos datos se presentan en (Hernández Díaz \& Orellana Molina, 2019; Núñez, 2014). En (Núñez, 2014) se encuentra un protocolo médico brasileño el cual es usado en el tratamiento fotodinámico de un equipo comercial que usa dos longitudes de onda denominado "Láser Duo", el cual aplica dosis similares al de la Tabla 6 dependiendo de la enfermedad a tratar.

Tabla 6. Plan dosificado de aplicación de energía.

\begin{tabular}{|c|c|}
\hline Efecto terapéutico deseado & Dosis recomendada $\left[\mathrm{J} / \mathrm{cm}^{2}\right]$ \\
\hline \multicolumn{2}{|l|}{ analgésico } \\
\hline - Dolor muscular & $2-4$ \\
\hline - $\quad$ Dolor articular & $4-8$ \\
\hline \multicolumn{2}{|l|}{ Antiinflamatorio } \\
\hline - $\quad$ Agudas & $4-6$ \\
\hline - crónicas & $4-8$ \\
\hline reparación tisular & $8-12$ \\
\hline
\end{tabular}

De la Tabla 6 se concluye que con el prototipo realizado se puede tener las dosificaciones recomendadas al aumentar el tiempo de aplicación de 3 a 4 minutos.

\subsubsection{Pruebas espectrales}

Las pruebas espectrales se las realizan basadas en la Norma InMetro 8164 (inmetro.gov.br, 2019), y utilizando el espectrómetro FLAME-S-VIS-NIR-ES de Ocean Optics, al cual tiene como aditamento una fibra óptica que facilita el direccionamiento de la luz hacia el sensor, proporcionando los datos adecuados del espectro de emisión. El espectrómetro se conecta al computador mediante comunicación serial como se muestra en la Figura 17 para mostrar el espectro de emisión en la pantalla.

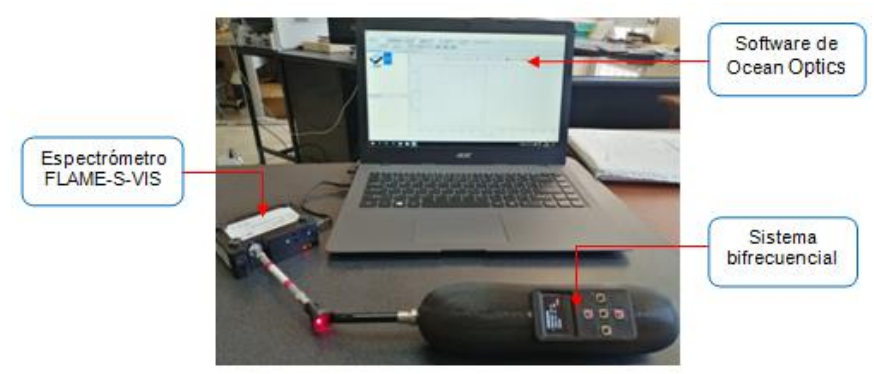

Figura 17. Adquisición de los espectros de emisión.

La prueba se lo realiza directamente con la luz generada por cada láser después de pasar por cada elemento óptico como lentes, espejo, espejo dicroico y fibra óptica los cuales interfieren en la medida distorsionando el espectro de emisión y la dispersión de la luz. Los resultados se presentan en las Figura 18 y Figura 19 los cuales corresponden al espectro de emisión de la luz rojo e infrarrojo respectivamente.

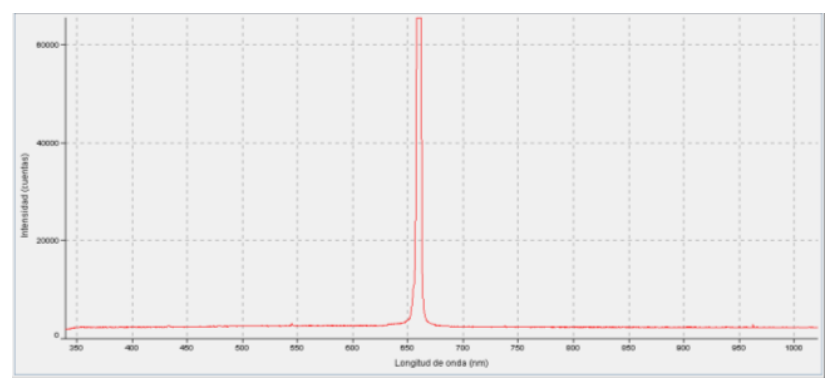

Figura 18. Espectro de emisión de la luz roja.

En la Figura 18 se observa que tiene un pico en los 660 (nm) con una dispersión de \pm 7 (nm).

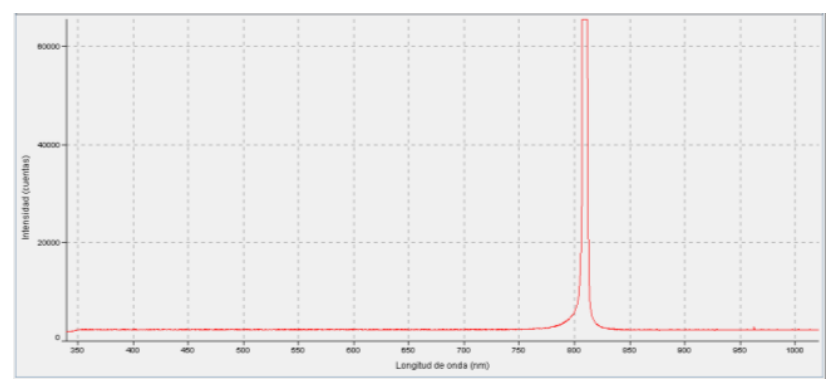

Figura 19. Espectro de emisión de la luz infrarroja.

En la Figura 19 se observa que tiene un pico en los 808 (nm) con una dispersión de $\pm 10(\mathrm{~nm})$.

\subsubsection{Pruebas térmicas}

Las pruebas térmicas del prototipo se las realiza de acuerdo con la Norma InMetro 8297 (inmetro.gov.br, 2019), usando un termómetro FLUKE de la serie VT, el cual es un termómetro visual de infrarrojo, que combina una medición de punto central con una imagen digital y una imagen superpuesta de mapa calorífico. En la Figura 20, se observa la pantalla del termómetro con los detalles que presenta en ella como es: la temperatura máxima y mínima, la hora y fecha, emisividad. La pantalla cuenta con un cursor que facilita la medición de la temperatura máxima y mínima.

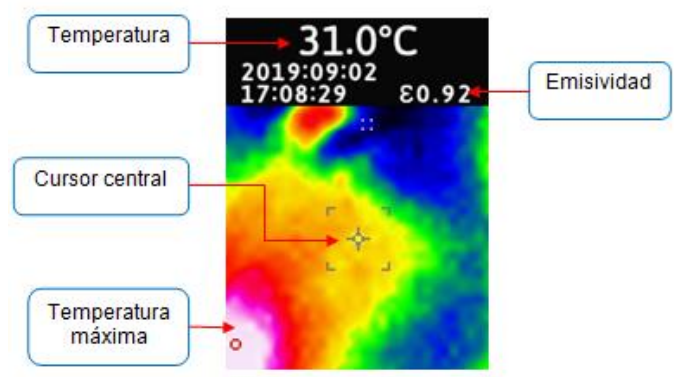

Figura 20. Pantalla del termómetro FLUKE VT04.

Las pruebas térmicas se realizaron activando cada láser a su máxima intensidad, después de 10 minutos de estar en funcionamiento se realiza la medición térmica en donde se tiene una temperatura máxima de $40\left({ }^{\circ} \mathrm{C}\right)$, la cual es generada por el regulador de voltaje de los láseres, si bien esta temperatura es alta no afecta al elemento ya que en las especificaciones técnicas del fabricante dice que la temperatura máxima que soporta es $120\left({ }^{\circ} \mathrm{C}\right)$, otra temperatura muy similar se registró en la resistencia de protección del láser de aproximadamente $38\left({ }^{\circ} \mathrm{C}\right)$, en el resto de elementos se 
mantenía una temperatura que oscilaba entre los 30 a $33\left({ }^{\circ} \mathrm{C}\right)$ la cual no se considera alta, en la Figura 21 se presenta las imágenes térmicas tomadas del prototipo.

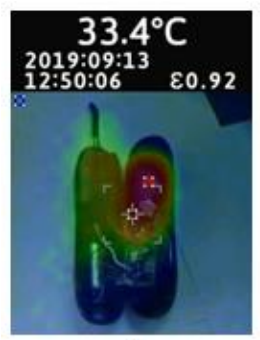

A

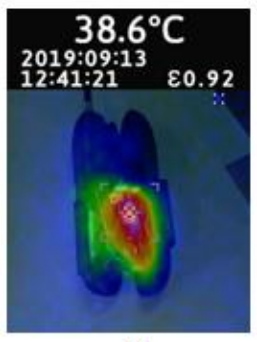

B

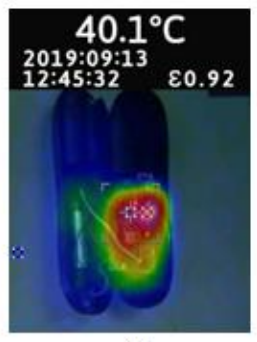

C
Figura 21. Pruebas térmicas.

Actualmente el Laboratorio de Aplicaciones Ópticas y UAVs se encuentra realizando los protocolos médicos y el registro sanitario del equipo para ser validado y poder ser usado en personas con todas las seguridades.

\section{CONCLUSIONES}

La batería de iones de litio al ser cargada mediante una fuente externa cuenta con protecciones que ayudan a que su vida útil se prolongue por más tiempo, para lo cual se tiene protecciones contra: sobrecarga, sobre descarga y calentamiento, que actúan de manera independiente ante cualquier evento anormal.

La implementación de filtros capacitivos para mejorar las señales en los sensores de voltaje y corriente utilizados, son de mucha importancia pues ayudan a tener una señal que no varíe en el tiempo, de esta manera no se pierde información al momento de ser procesada por la tarjeta de desarrollo mediante los puertos análogos.

La selección del circuito integrado LM358 como sensor de voltaje se lo hace por las características propias del integrado como: impedancia de entrada alta, presenta poco ruido de entrada, únicamente necesita una fuente de alimentación. Por su tamaño, el integrado contiene dos amplificadores operacionales suficientes para el sistema.

Al programar los registros del OCR0, se tiene dos señales modulares, las cuales tienen una frecuencia de $3 \mathrm{KHz}$ y se activan dependiendo del láser que se va a utilizar. Estas señales de control se envían hacia los transistores para que trabajen en corte y saturación a la frecuencia mencionada, de esta manera se regula el voltaje para tener varios niveles de iluminación asociado directamente con la irradiancia, es decir mientras más grande sea la relación de trabajo más alto será el nivel de irradiancia.

El modelo óptico está basado en arreglos de espejos y lentes, los cuales constituyen una manera práctica para direccionar los haces de luz hacia un mismo punto, dando como resultado un espectro de emisión continuo, conservando los principios físicos de la luz láser.
En lo que a los conectores concierne se utilizó conectores tipo Molex con ciertas modificaciones ya que por sus características constructivas ocupan mucho espacio, se recortó la base de estos liberando así un espacio de $3 \mathrm{~mm}$, al alterar el diseño de los conectores se pierden las muescas de seguridad que tienen, para saber cómo va conectado se pinta una de las caras de cada conector dirigida hacia afuera de la PCB.

El prototipo al poseer una batería de iones de litio, una pantalla de visualización y botones lo convierte en un equipo portátil con un tiempo de autonomía de 4 a 5 horas funcionando a su máxima irradiancia $\left(25 \mathrm{~mW} / \mathrm{cm}^{2}\right)$ para el láser rojo y $(15$ $\mathrm{mW} / \mathrm{cm}^{2}$ ) para el láser infrarrojo.

\section{REFERENCIAS}

Calabrese, G. (2011, agosto). Láser y luz pulsada intensa. Revista Médica de Uruguay, p. 8.

Carrasco Paz y Miño, M. P. (2018). Eficacia de la Terapia Fotodinámica con láser de baja potencia, en tratamiento periodontal básico, en estudio clínico, Randomizado (Tesis de pregrado). Universidad San Francisco de Quito, Quito, Ecuador.

De Paula Eduardo, C., Aranha, A. C. C., Simões, A., BelloSilva, M. S., Ramalho, K. M., Esteves-Oliveira, M., ... Tunér, J. (2013). Laser treatment of recurrent herpes labialis: A literature review. Lasers in Medical Science. Doi: 29. 10.1007/s10103-013-1311-8.

DeltaOHM. (2019, septiembre 30). DeltaOHM [Html]. Recuperado el 30 de septiembre de 2019, de DeltaOHM-Manual de instrucciones.

España-Tost, A. J., Arnabat-Domínguez, J., Berini-Aytés, L., \& Gay-Escoda, C. (2004). Aplicaciones del láser en Odontología. RCOE, 9(5). 497-511.

González, B. M., Hernández, A., \& Estevez, A. (2008). Tratamiento del herpes simple labial con láser de baja potencia. Colombia Médica, 39(2), 175-181.

Hamblin, M. R. (2013, febrero). Mechanisms Of Low Level Light Therapy [Asp]. Recuperado el 8 de agosto de 2019, de Massachusetts General Hospital.

Hamblin, M. R., \& Huang, Y.-Y. (2013). HandBook of Photomedicine (1st ed.).

Hernández Díaz, A., \& Orellana Molina, A. (2019, septiembre 22). Dosimetría en láseres de baja potencia [Medica]. Recuperado el 22 de septiembre de 2019, de Centro de Aplicaciones Tecnológicas y Desarrollo Nuclear (CEADEN).

inmetro.gov.br. (2019a, octubre 15). Norma de Espectrometria [Html]. Recuperado el 15 de octubre de 2019, de InMetro. 
Marotti, J., Aranha, A. C. C., Eduardo, C. D. P., \& Ribeiro, M. S. (2009). Photodynamic Therapy Can Be Effective as a Treatment for Herpes Simplex Labialis. Photomedicine and Laser Surgery, 27(2), 357-363. doi: 10.1089/pho.2008.2268.

Núñez, S. C. (2014). Protocolo de laser terapia e terapia fotodinâmica laser duo. MMO tecnoogia para a saúde, 2,57 .

Pacheco Rodriguez, L. (2013). Desarrollo de un sistema de irradiación para Terapia Fotodinámica Intersticial (Tesis de grado). Instituto Politécnico Nacional, México D.F., México.

Pacheco Rodriguez, L., Isakina, S. S., De la Rosa Vazquez, J. M., \& De la Cadena Perez Gallardo, A. (2014). Laser Diode System For Interstitial Photodynamic Therapy. IEEE Latin America Transactions, 12(4), 574-579.

Pacho Saavedra, J. A., \& Piñol Jiménez, F. N. (2006). Lesiones bucales relacionadas con las enfermedades digestivas. Revista Cubana de Estomatología, 43(3).

Ponce Chango, C. D. (2018). Diseño e implementación de un prototipo para adquisición de imágenes de fluorescencia a ser usado en el diagnóstico de cáncer de piel de tipo no melanoma. (Tesis pregrado). Escuela Politécnica Nacional, Quito, Ecuador.

Schindl, A., \& Neumann, R. (1999). Low-Intensity Laser Therapy is an Effective Treatment for Recurrent Herpes Simplex Infection. Results from a Randomized Double-Blind Placebo-Controlled Study. Journal of Investigative Dermatology, 113(2), 221-223.

Stolik, S., Delgado, J. A., Perez, A., \& Anasagasti, L. (2000). Measurement of the penetration depths of red and near infrared light in human "“ex vivo" tissues. Journal of photochemistry and photobiology. B, Biology. 57. 903. Doi: 10.1016/S1011-1344(00)00082-8

tecnica1lomas.com. (2019, julio 26). Proteus 8.6 [Html]. Recuperado el 26 de julio de 2019, de Tecnica1lomas.

Tellez Tielves, Norma de la Caridad, Pérez Hernández, Leyda Yenima, Rodríguez Ávila, Jesús, Travieso Pérez, Maritza, \& López Blanco, María de las Nieves. (2013). Efectividad del tratamiento con radiación láser de baja potencia en la estomatitis aftosa recurrente. Revista de Ciencias Médicas de Pinar del Río, 17(5), 40-50. Recuperado en 13 de mayo de 2020.

Tutivén Abad, C. F. (2017). Estudio clínico, randomizado, controlado para evaluar la eficiencia de la Terapia fotodinámica con láser de baja potencia como coadyuvante de la Terapia básica periodontal comparada con la Terapia básica periodontal sola. (Tesis postgrado). Universidad San Francisco de Quito. Quito, Ecuador.
Valiente Zaldívar, C. J., \& Garrigó Andreu, M. I. (1997). Laser blando en puntos de acupuntura para el tratamiento de enfermedades bucales. Revista Cubana de Estomatología, 34, 5-10.

\section{BIOGRAFÍAS}

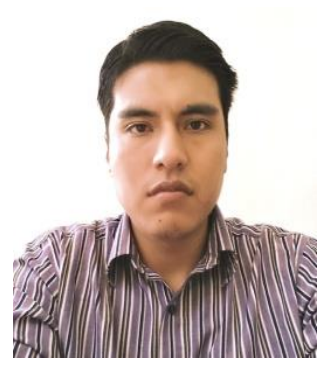

Santiago Matango, Nació el 22 de mayo de 1992 en Quito-Ecuador, sus estudios secundarios los realizó en la unidad educativa "Juan de Salinas", donde obtuvo el título de bachiller en la especialidad Físico Matemático. Estudió en la Escuela Politécnica Nacional, obteniendo el título de Ingeniero en Electrónica y Control, actualmente se encuentra desarrollando proyectos independientes en áreas a fines.

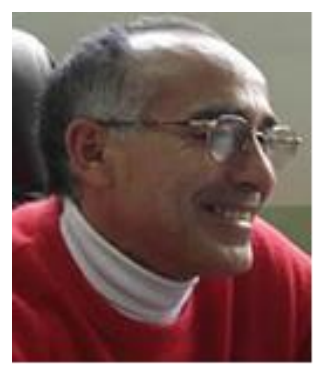

Eduardo Ávalos es Doctor en Física de la Pontificia Universidad Católica de Río de Janeiro (PUC-EPN) e ingeniero de la Escuela Politécnica Nacional (EPN). Coordinador de la Red Fotónica Brasil - Ecuador. Premio EPN a la Mejor Investigación 2019, y reconocimiento a mejor profesor. Fue Director de Investigación de la EPN, Decano de la Facultad de Ciencias, y director de la Escuela de Ciencias de la Escuela Politécnica Nacional, profesor invitado en la Universidad Católica de Rio de Janeiro, ESP, UFSaoCar Sao Paulo Brasil, coordinador de la REDU. Actualmente es profesor de la Escuela Politécnica Nacional a tiempo completo y se encuentra a cargo del laboratorio de UAV's y Aplicaciones Ópticas. 